\title{
Point mutations within the streptococcal regulator of virulence (Srv) alter protein-DNA interactions and Srv function
}

\author{
Christopher D. Doern, Robert C. Holder and Sean D. Reid
}

Correspondence

Sean D. Reid

sreid@wfubmc.edu

Received 21 September 2007

Revised 22 January 2008

Accepted 25 March 2008

\author{
Department of Microbiology and Immunology, Wake Forest University School of Medicine, Winston- \\ Salem, NC 27157, USA
}

\begin{abstract}
Group A Streptococcus (GAS) possesses a complex regulatory system enabling the organism to colonize a range of physiologically distinct host sites. Within this network of regulators is the streptococcal regulator of virulence (Srv). Srv is a member of the CRP/FNR family of transcriptional regulators and is most similar to pleiotropic regulatory factor A (PrfA), a positive regulator of virulence in Listeria monocytogenes. Members of this family possess a characteristic C-terminal helix-turn-helix motif $(\mathrm{HTH})$ that facilitates binding to DNA targets. Genome scanning identified four targets in GAS that were similar to the consensus DNA target recognized by PrfA. Furthermore, previous amino acid sequence alignments identified conserved residues within the Srv HTH which are necessary for function in PrfA and CRP. Here we investigated the ability of Srv to interact with DNA and evaluated the role of the HTH in this interaction. Purified recombinant Srv (rSrv) was found to co-purify with an untagged form of Srv. Glutaraldehyde cross-linking and gel-filtration chromatography indicated that this co-purification is likely due to the ability of Srv to oligomerize. Electrophoretic mobility shift assays (EMSAs) demonstrated that rSrv retarded the mobility of DNA targets and a supershift analysis confirmed the observation was rSrv-dependent. Competition EMSA indicated that rSrv had a higher relative affinity for the DNA targets studied than non-specific DNA. Site-directed mutagenesis of residues predicted to be in or near the HTH resulted in a decrease or abrogation of DNA binding. Complementation of MGAS5005 $\Delta$ srv with one of these site-directed mutants failed to restore wild-type SpeB activity. Taken together, these data suggest that the Srv HTH is necessary for DNA binding and Srv function.
\end{abstract}

\section{INTRODUCTION}

Group A Streptococcus (GAS) is a Gram-positive human pathogen that has evolved to colonize a diverse group of physiologically distinct host sites resulting in diseases that range from mild (pharyngitis, impetigo) to severe (necrotizing fasciitis, streptococcal toxic shock-like syndrome) (Cunningham, 2000; Musser \& DeLeo, 2005). To this end, the bacterium possesses a reticulate regulatory network consisting of up to 13 two-component regulators and as many as 100 single-response or stand-alone regulators (Graham et al., 2002). While many of these elements have only been identified in silico, all regulators that have been studied in vivo appear to be critical for GAS virulence (Banks et al., 2004; Beres et al., 2004; Ferretti et al., 2001; Kreikemeyer et al., 2003; Smoot et al., 2002).

Abbreviations: CRP, cAMP receptor protein; EMSA, electrophoretic mobility shift assay; FNR, fumarate and nitrate reduction regulator; GAS, group A Streptococcus; HTH, helix-turn-helix.
Within the GAS regulatory network is SPy1857 or the streptococcal regulator of virulence (Srv). A serotype M1 strain lacking srv (MGAS5005 $\Delta r v$ ) was significantly attenuated in a murine model of systemic infection, leading to the hypothesis that Srv is a critical factor in GAS virulence (Reid et al., 2004). Amino acid alignment of clinical isolates representing 12 GAS serotypes revealed a lone amino acid substitution at position 217 in a serotype M6 strain (Reid et al., 2004). Thus, there is strong evidence of selective pressure to maintain Srv function. In testing Srv-mediated regulation, microarray analysis showed that significant differences exist between the expression profiles of MGAS5005 $\Delta r r v$ and the parental serotype M1 strain (MGAS5005). Given that our study was designed to assess the regulatory potential of Srv, we elected to use a 1.5-fold threshold. Results indicated that $21 \%$ of GAS genes are downregulated in the isogenic srv mutant. While loss of $s r v$ resulted in broad downregulation, only seven genes were upregulated in MGAS5005 ssrv (Reid et al., 2006). Taken together, these results suggest that Srv is an important transcriptional regulator required for GAS survival in the 
human host. Furthermore, obtaining a deeper understanding of Srv function may provide novel insights into GAS pathogenesis and potential therapeutic targets.

Sequence comparisons predicted that Srv is a member of the cAMP receptor protein (CRP)/fumarate and nitrate reduction regulator (FNR) family of transcriptional regulators. CRP/FNR is a large family consisting primarily of positive transcriptional regulators and is named for its initial members discovered in Escherichia coli. Members of this family are generally found in Gram-negative organisms, with only a few studied Gram-positive examples. The family is defined by two conserved motifs: a $\beta$-roll region implicated in allosteric effector molecule binding and a helix-turn-helix (HTH) DNA-binding region. Computer modelling (http://expasy.org/sprot/) predicted that Srv, a 240 amino acid protein of $27.9 \mathrm{kDa}$, possessed both an $\mathrm{N}$-terminal $\beta$-roll structure and a C-terminal HTH motif (Reid et al., 2004); however, it has yet to be shown that either motif is functional.

Within the CRP/FNR family, the regulator with the most sequence similarity to Srv is pleiotropic regulatory factor A (PrfA) of Listeria monocytogenes, which demonstrates $27 \%$ identity and $53 \%$ similarity. PrfA has been shown to differentially activate transcription of virulence genes found on a $9 \mathrm{~kb}$ pathogenicity island (Bubert et al., 1999; Mengaud et al., 1991). Electrophoretic mobility shift assays (EMSAs) and transcription studies indicate that the stringency of PrfA-mediated control is directly related to the binding affinity for the PrfA-box (Bohne et al., 1996; Sheehan et al., 1995). The PrfA-box is a cis-acting element located upstream of genes under PrfA-mediated control. Specifically, these sequences are located approximately $41 \mathrm{bp}$ upstream of given transcriptional start sites and are $14 \mathrm{bp}$ of dyad symmetry (Freitag et al., 1993). The consensus PrfA-box is a perfect palindrome and is bound with highest affinity (Dickneite et al., 1998). As sequence degenerates from the consensus, PrfA affinity decreases and consequently so does the tightness of PrfA-mediated control (Bockmann et al., 2000; Dickneite et al., 1998; Dramsi et al., 1993; Freitag et al., 1993; Herler et al., 2001; Mengaud et al., 1989; Vega et al., 2004).

Unlike prfA, srv was identified by a GAS genome search rather than in association with a distinct phenotype. As a result, no targets under direct Srv-mediated control were immediately obvious upon discovery. Thus, it is unclear if Srv has a focused number of targets, or if it assists in the transcription of a diverse array of genes as suggested by microarray. Based on the sequence similarity between the Srv and PrfA HTH regions, we hypothesized that Srv may bind DNA sequences similar to the PrfA-box. Four genes within the GAS genome were identified as having PrfAbox-like sequences in their promoter regions (Reid et al., 2004). In mutant strains lacking srv, real-time RT-PCR analyses showed reduced transcript levels for these genes, suggesting that they are under Srv-mediated regulation (Reid et al., 2004). In the present study we examined the role of the Srv HTH in DNA binding and in Srv function.

\section{METHODS}

Bacterial strains. MGAS5005 was isolated from a case of invasive GAS disease and has been the focus of numerous studies (reviewed by Reid et al., 2001). The MGAS5005 isogenic srv mutant strain was generated by allelic replacement (Reid et al., 2004). The isogenic mutant strain lacking srv was complemented using shuttle vector pIA $\beta 8$ (Pérez-Arellano et al., 2001). Wild-type srv coding sequence was amplified from purified MGAS5005 genome using primers AAAAGGTACCTGTTACTATCAAAGGGCATTAGC and AAGAATTCGGAGTTTCCTTTCTAGTGGC. Site-directed mutant $s r v_{\mathrm{R} 207 \mathrm{G}}$ was amplified from plasmid pSDRSrv ${ }_{\text {R207G }}$ using primers AAAAAGAATTCTTAGAAAACGATATTAT and AAAAGGTACCTTACTATCAAAGGGCATTAGCAAATGTTAATTTCTCTTGA. The respective coding sequences were directionally cloned into pIA $\beta 8$ and transformed into E. coli NovaBlue cells according to the manufacturer's specifications (Novagen). Approximately $15 \mu \mathrm{g}$ of purified plasmid was incubated with $50 \mu \mathrm{l}$ of competent MGAS5005 $\Delta s r v$ cells on ice for $10 \mathrm{~min}$. Cells were then electroporated in a prechilled $0.1 \mathrm{~cm}$ electroporation cuvette $(400 \Omega, 25 \mu \mathrm{F}, 1.8 \mathrm{kV})$. Immediately after electroporation $1 \mathrm{ml}$ THY medium (Becton Dickinson) was added to the cuvette. Cells were incubated at $37{ }^{\circ} \mathrm{C} / 5 \% \mathrm{CO}_{2}$ for $2 \mathrm{~h}$ and then plated on selective medium containing $2 \mu \mathrm{g}$ chloramphenicol ml $\mathrm{ml}^{-1}$. Gene expression and protein production were assayed by real-time reverse transcriptase PCR and Western immunoblot analysis, respectively (data not shown).

Protein purification. For expression and purification of recombinant protein, srv was cloned into expression vector pET32A (renamed pSDRsrv) and transformed into E. coli NovaBlue (Novagen). Strains containing pSDRsrv were selected with $50 \mu \mathrm{g}$ ampicillin $\mathrm{ml}^{-1}$. Recombinant ( His $_{6}$-tagged) Srv protein was purified from E. coli protein expression strain Origami(DE3) (Novagen). Srv production was induced by $1 \mathrm{mM}$ IPTG in a bacterial culture at $\mathrm{OD}_{600} 0.5$. After induction the culture was incubated for an additional $3 \mathrm{~h}$ and harvested by centrifugation. Cells were lysed in BugBuster Protein Extraction Reagent (Novagen). Cell debris was pelleted and discarded. Remaining solution was passed over an Ni-NTA HisBind resin column (Novagen). Bound protein was washed and eluted according to the manufacturer's specifications. Collected protein was dialysed at $4{ }^{\circ} \mathrm{C}$ in $20 \mathrm{mM}$ Tris/ $\mathrm{HCl} \mathrm{pH} 8.0$ twice for $2 \mathrm{~h}$ with a third and final dialysis overnight. Purified samples were separated on $15 \%$ SDSpolyacrylamide gel for $35 \mathrm{~min}$ at $200 \mathrm{~V}$ and stained in Coomassie blue for analysis.

Electrophoretic mobility shift assay (EMSA). PCR reactions with fluorescein (FAM)-labelled primers were used to generate fluorescent DNA probes. PCR products were separated on $3 \%$ agarose gels for $1 \mathrm{~h}$ at $100 \mathrm{~V}$. Gel extraction was used to separate PCR product from primer. Concentrations of $6.7 \mathrm{nM}$ and $8.7 \mathrm{nM}$ probe were used in binding and competition EMSA experiments, respectively. Binding reactions were all conducted at room temperature for $20 \mathrm{~min}$ in EMSA binding buffer (10 mM HEPES pH 7.6, 1 mM EDTA, 2 mM DTT, $50 \mathrm{mM} \mathrm{KCl}, 0.05 \%$ Triton X-100, $0.07 \mathrm{mg}$ poly d(I-C) $\mathrm{ml}^{-1}$ and $5 \%$ glycerol). Bound products were separated from free DNA on $4 \%$ native polyacrylamide gel in $1 \times$ Tris/borate/EDTA (TBE). Gels were run at $200 \mathrm{~V}$ for $90 \mathrm{~min}$ at $4{ }^{\circ} \mathrm{C}$. As a negative control, slr, which encodes a streptococcal surface protein not expected to exhibit DNAbinding activity, was cloned into pET32A, purified, and included in an EMSA reaction. No DNA binding was observed with this control (data not shown). Immediately following electrophoresis, gel images were captured on a Typhoon 8600 variable-mode scanner and viewed by ImageQuant version 5.2 software (Molecular Dynamics). Relative affinity of DNA binding $\left(R_{\mathrm{A}}\right)$ was calculated using quantitative EMSA. The dynamic range of protein concentration used in these experiments was 0 to $30.6 \mu \mathrm{M}$. $R_{\mathrm{A}}$ was defined as the amount of rSrv required to shift $50 \%$ of the free DNA as described by Riggs et al. 
(1971). ImageQuant v. 5.2 was used to calculate percentage shift via pixel densitometry. Density of unbound DNA was computed and used in the following equation to determine percentage shift: Percentage shift $=[($ Total free DNA-Experimental free DNA $) /$ Total free DNA] $\times 100$.

In this equation 'Total free DNA' is equal to the value computed for unbound DNA in negative control lanes. All EMSA experiments were repeated at least three times. $R_{\mathrm{A}}$ values are reported as an average of experiments conducted with each target.

Sequence analysis. Sequencing was done by Macrogen (Seoul, South Korea). Consensus sequences were compiled using SeqMan II (DNASTAR). The consensus sequences were identified and analysed using EditSeq (DNASTAR).

Glutaraldehyde cross-linking. Untagged Srv in $20 \mathrm{mM}$ Tris/ $\mathrm{HCl}$ $\mathrm{pH} 8.0$ was treated with the protein cross-linking agent glutaraldehyde. The reaction mixture contained $7.8 \mathrm{nM}$ untagged Srv and $1.0 \mathrm{mM}$ glutaraldehyde in a total volume of $20 \mu \mathrm{l}$. Reactions were conducted at room temperature. Following the glutaraldehyde reaction, $66 \mathrm{mM}$ sodium borohydride was added and incubated on ice for $10 \mathrm{~min}$. The reaction was quenched with $230 \mathrm{mM}$ Tris. Denaturing loading dye was added to each sample and boiled for 5 min prior to analysis on $15 \%$ SDS-polyacrylamide gel with Coomassie blue staining.

Gel-filtration chromatography. Analytical gel-filtration chromatography was done on a Sephacryl S-200 High Resolution HiPrep 16/60 column using the AKTA FPLC system (GE Healthcare), preequilibrated with $20 \mathrm{mM}$ Tris/ $\mathrm{HCl} \mathrm{pH} 8.0$ and calibrated with molecular mass standards blue dextran, conalbumin $(75 \mathrm{kDa})$, ovalbumin $(43 \mathrm{kDa})$, carbonic anhydrase $(29 \mathrm{kDa})$ and RNase A $(13.7 \mathrm{kDa})$ (GE Healthcare). Protein samples were run in $20 \mathrm{mM}$ Tris/HCl $\mathrm{pH}$ 8.0. The size of the Srv dimer was determined by plotting the $K_{\mathrm{av}}$ versus the log of the molecular mass. The $K_{\mathrm{av}}$ is defined by the equation $K_{\mathrm{av}}=\left(V_{\mathrm{e}}-V_{0}\right) /\left(V_{\mathrm{c}}-V_{0}\right)$, where $V_{\mathrm{e}}$ is the elution volume of the species, $V_{0}$ is the void volume, and $V_{c}$ is the geometric column volume. The $V_{0}$ was determined to be $35.9 \mathrm{ml}$ with the high-molecular-mass marker blue dextran, while the $V_{c}$ was determined to be $120 \mathrm{ml}$. Eluted volumes were concentrated by vacuum centrifugation and analysed via Western blotting using polyclonal anti-Srv antibody (Rockland).

Enterokinase cleavage. The $\mathrm{N}$-terminal recombinant tag of purified rSrv was cleaved using the Tag.off rEK Cleavage Capture kit according to the manufacturer's specifications (Novagen). Following cleavage and capture, sample was dialysed at $4{ }^{\circ} \mathrm{C}$ in $20 \mathrm{mM}$ Tris/ $\mathrm{HCl}$ pH 8.0 twice for $2 \mathrm{~h}$ with a third and final dialysis overnight.

Casein agar plate assay. The proteolytic activity of SpeB was determined as described by Hynes \& Tagg (1985). Briefly, MGAS5005, MGAS5005 $\Delta s r v, \quad$ MGAS5005 $\Delta r v($ pIA $\beta 8-s r v)$ and MGAS5005 $\Delta r r\left(\mathrm{pIA} \beta 8-\operatorname{srv}_{R 207 G}\right)$ were stab inoculated into ToddHewitt agar plates containing $3 \%$ skim milk (Difco), and incubated anaerobically at $37{ }^{\circ} \mathrm{C}$ for approximately $18 \mathrm{~h}$ in an anaerobic jar (Anaerocult) (Hynes \& Tagg, 1985). A zone of translucence around the stab site indicated the presence of caseinolytic activity.

\section{RESULTS}

\section{Purification of rSrv}

To purify Srv, srv coding sequence was amplified and directionally cloned into expression vector pET32A
(Novagen), which contains a $\mathrm{His}_{6}$ and an S-tag fusion to facilitate purification. In addition, it possesses a thioredoxin tag to promote protein solubility. SDS gel analysis of the purification product revealed two species, one of $\sim 45 \mathrm{kDa}$ and one of $\sim 26 \mathrm{kDa}$ (Fig. 1). MALDI-TOF analysis of rSrv purifications confirmed the size of these species at $44.84 \mathrm{kDa}$ and $25.58 \mathrm{kDa}$, respectively. Subsequent N-terminal sequencing identified the $45 \mathrm{kDa}$ product as recombinant tagged Srv (10 correct N-terminal amino acids). $\mathrm{N}$-terminal sequencing was unable to identify the lower band, consistent with a chemical modification blocking the $\mathrm{N}$ terminus. In the cloning process, the native translational start site of Srv was left intact. We hypothesize that a proportion of Srv was translated from the native start site, producing protein without the $\mathrm{N}$-terminal tag.

\section{rSrv demonstrates the ability to oligomerize}

Untagged Srv demonstrated no affinity for the nickel column used in purification; this suggested that copurification of untagged Srv was due to oligomerization with rSrv. CRP/FNR family members CRP and PrfA have both been shown to dimerize via crystallographic analyses, lending further strength to this hypothesis (Eiting et al., 2005; Parkinson et al., 1996; Passner et al., 2000; Velge et al., 2007). CRP has been shown to form a homodimer in which each monomer interacts with a half-site of the $14 \mathrm{bp}$ palindromic consensus binding sequence (Parkinson et al., 1996; Schultz et al., 1990, 1991; Weber \& Steitz, 1987). Given the sequence similarity between Srv and CRP/FNR family members, and the observation made during purification of rSrv, we hypothesized that Srv might form oligomers. To test this hypothesis we conducted

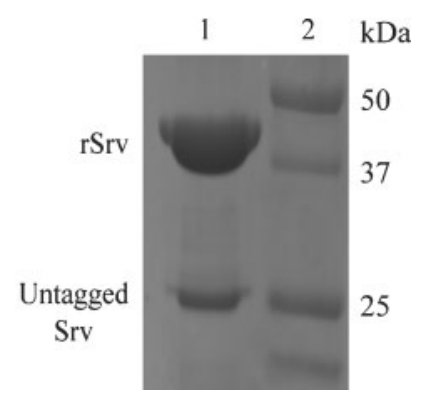

Fig. 1. Co-purification of two species of Srv. For expression and purification of recombinant protein, srv was cloned into expression vector $\mathrm{pET} 32 \mathrm{~A}$ to generate $\mathrm{pSDR}$ srv. The native translational start and termination sites of Srv were left intact. Following overexpression and purification on a Ni-NTA column, SDS-PAGE revealed the presence of two proteins (lane 1). One corresponded to the predicted weight for recombinant Srv (rSrv), $\sim 45 \mathrm{kDa}$, while the other migrated to the predicted weight of untagged Srv, $\sim 26 \mathrm{kDa}$ (the thioredoxin, $\mathrm{His}_{6}, \mathrm{~S}$ protein-tag adds $\sim 17 \mathrm{kDa}$ ). Lane 2 contains a protein standard ladder with molecular masses indicated to the right. 
glutaraldehyde cross-linking. Glutaraldehyde irreversibly cross-links proteins and therefore oligomeric products can be analysed with denaturing PAGE. To exclude the possibility that the recombinant tag contributes to oligomerization, untagged Srv, generated from enterokinase cleavage reactions, was used in cross-linking experiments. In a time-dependent manner, glutaraldehyde crosslinking produced an increasing concentration of oligomeric species (species II) migrating slightly slower than the $75 \mathrm{kDa}$ standard (Fig. 2a). The predicted sizes of an Srv dimer and trimer are $\sim 56 \mathrm{kDa}$ and $\sim 84 \mathrm{kDa}$, respectively. The effects of glutaraldehyde cross-linking on the migration of Srv through a denaturing polyacrylamide gel are unclear. Thus, while we cannot conclusively state that Srv forms a dimer or trimer, these results suggest that

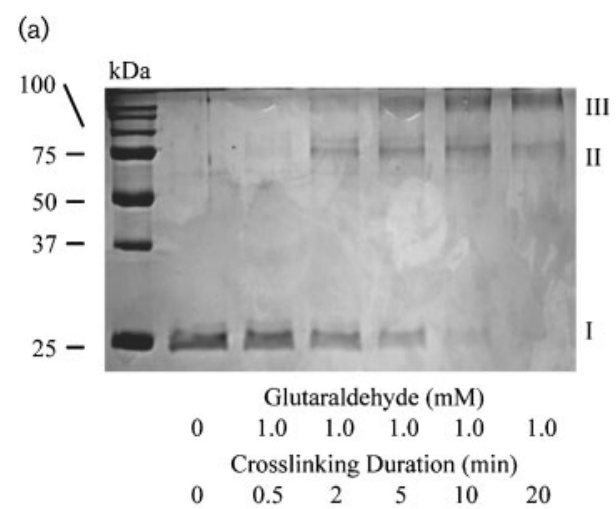

(b)

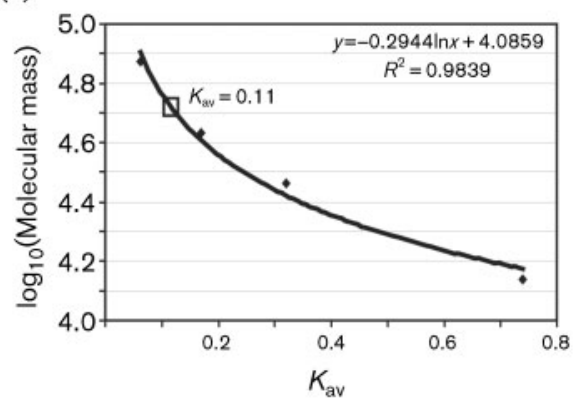

Fig. 2. Oligomerization of Srv demonstrated via glutaraldehyde cross-linking (a) and gel filtration chromatography (b). (a) rSrv was subjected to enterokinase cleavage to remove the $\mathrm{His}_{6}$-tag prior to cross-linking. Glutaraldehyde was added to $6.8 \mathrm{nM} \mathrm{Srv}$ for up to $20 \mathrm{~min}$ at room temperature. SDS analysis indicated that the monomer (species I, $27.9 \mathrm{kDa}$ ) became incorporated in a band (species II) migrating near $75 \mathrm{kDa}$ suggestive of a Srv dimer $(\sim 55.8 \mathrm{kDa})$ or trimer $(\sim 83.7 \mathrm{kDa})$. A higher-order multimer is also formed (species III) which is likely due to non-specific cross-linkage by glutaraldehyde. (b) A standard curve was generated based on the elution of the following protein standards: RNase A (13.7 kDa), carbonic anhydrase (29 kDa), ovalbumin $(43 \mathrm{kDa})$ and conalbumin $(75 \mathrm{kDa})$. Srv eluted in a volume with a $K_{\mathrm{av}}$ of 0.11 , which calculated to a size of $54.4 \mathrm{kDa}$, indicative of a dimer. oligomerization does occur. As stated above, MALDITOF analysis indicated our protein was pure, eliminating the possibility of another protein being present. Importantly, at $10 \mathrm{~min}$ the majority of the protein appeared to be in an oligomerized state (species II and III) as evidenced by the nearly complete loss of monomeric species (species I). Additionally, from 2 to $10 \mathrm{~min}$ no increase in dimer/trimer formation was observed; however, higher-order multimer (species III) formation increased over this time. It is not clear if species III is the result of specific interactions among Srv molecules, but non-specific cross-linkage by glutaraldehyde resulting in such higherorder multimers has been observed (Kuusinen et al., 1999).

Size-exclusion chromatography was employed to further analyse the oligomeric state of purified rSrv and determine whether complexes observed in glutaraldehyde crosslinking were dimer or trimer species. To exclude the possibility that the recombinant tag was playing a role in oligomerization we analysed untagged Srv $(27.9 \mathrm{kDa})$. The Srv elution fraction had a $K_{\mathrm{av}}$ of 0.11 , which correlated with $\sim 54.4 \mathrm{kDa}$, the approximate size of a dimer (Fig. 2b). An independent experiment with purified rSrv (tagged, $\sim 45 \mathrm{kDa}$ ) yielded an elution fraction with a $K_{\mathrm{av}}$ of 0.052 , which, when extrapolated, corresponds to a predicted dimer weight of $\sim 90 \mathrm{kDa}$. These data confirm that Srv is capable of dimerization and suggest that the species identified in glutaraldehyde cross-linking was representative of a dimer and not a trimer.

\section{EMSAs demonstrate that purified rSrv binds PrfA- box-like sequences in the presence of non- specific competitor DNA}

Srv belongs to the CRP/FNR family of transcriptional regulators. Members of this family have been shown to mediate targeted regulatory effects through DNA-binding interactions. To test the hypothesis that Srv functions as a DNA-binding protein we selected four GAS targets previously identified to contain PrfA-box-like sequences (Fig. 3a) (Reid et al., 2004). These targets are designated spy0044, spy0285, spy0714 and slr and are located upstream of their corresponding genes spy0044 (zinccontaining dehydrogenase), spy0285 (ATP-binding protein), spy0714 (zinc-binding protein AdcA) and slr (streptococcal leucine-rich protein). In previous work, real-time RT-PCR analyses showed that the transcript level of all four genes was decreased in the srv isogenic mutant (Reid et al., 2004). Target DNA was generated using 5' fluorescein-labelled primers. Each primer set generated an approximately 105 bp fluorescent probe with a PrfA-boxlike sequence located in the centre of the fragment. Addition of rSrv retarded the electrophoretic mobility of each target, indicating that DNA binding had occurred (Fig. 3b). Several resolvable bands are observed. One interpretation of this result is that different numbers of Srv molecules are attached to the DNA fragments. This could result from binding to different sites along the DNA or Srv 
(a)

\section{L.m. PrfA-Box TTAACANNTGTTAA}

slr 5' - ACTTAAC-CA-GTTAAAT

spy0285 5' - GTTTAAC-TT-GTTAAAG

spy0044 5' - AATTAAC-CA-GTTAAGT

spy0714 5' - TTTTAAC-CA-GTTAATA

(b) spy0044

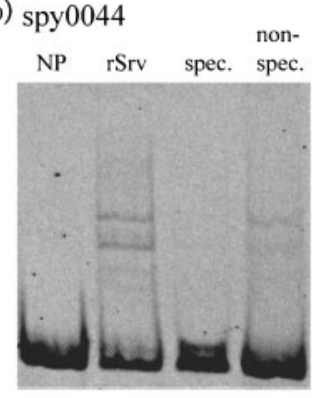

spy0285

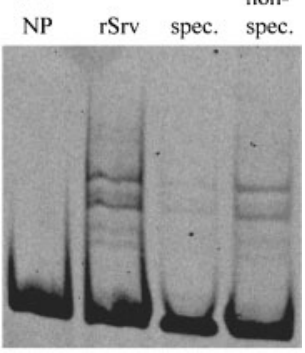

spy0714

slr

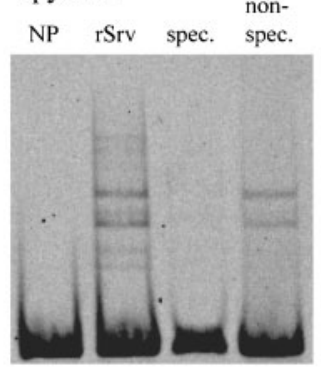

NP rSry non-

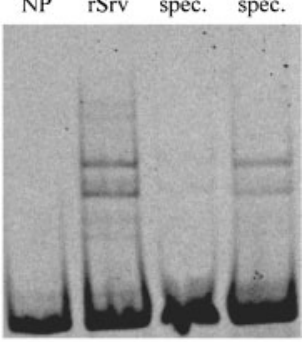

(c)

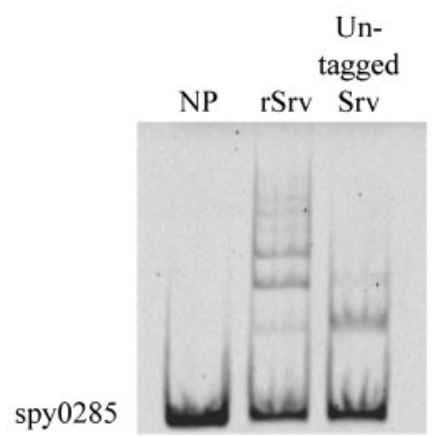

multimers with different numbers of molecules. This result has been seen and similar explanations suggested for protein-DNA interactions mediated by Staphylococcus aureus SarA and Pseudomonas aeruginosa AlgZ (Morfeldt et al., 1996; Ramsey et al., 2005; Rechtin et al., 1999). Our cross-linking data support the hypothesis that different Srv multimers are responsible for the different resolvable bands (more in Discussion).

To determine if Srv demonstrates a higher affinity for the four PrfA-box-like targets than for non-specific DNA, we conducted competition EMSAs utilizing herring-sperm DNA as a non-specific competitor (Blevins et al., 1999; Bockmann et al., 1996, 2000; Vega et al., 1998). Unlabelled PrfA-box-like targets served as specific competitors and herring-sperm DNA was the non-specific competitor. Standard competition binding reactions were conducted
Fig. 3. Purified rSrv retards the mobility of GAS DNA fragments containing PrfA-box-like sequences. (a) Scanning of the GAS M1 genome (SF370) identified four genes possessing PrfA-box-like sequences located in the vicinity of their promoter regions. Alignment of each sequence is shown with the $L$. monocytogenes PrfA-box consensus sequence in bold. (b) To test rSrv binding, $8.67 \mathrm{nM}$ of $\sim 105 \mathrm{bp}$ FAM-labelled PCR products were used as probes in EMSA. EMSA competition binding reactions were completed for each probe. Reactions without rSrv (NP) were used as a negative control. As a positive control, $4.2 \mu \mathrm{M}$ purified rSrv was used in the absence of competitor DNA. Specific competition (spec.) was studied by combining $4.2 \mu \mathrm{M}$ rSrv, probe and $51.5 \mu \mathrm{M}$ unlabelled probe ( 6000-fold excess). Non-specific (non-spec.) competition was studied by combining $4.2 \mu \mathrm{M}$ rSrv, probe and $51.5 \mu \mathrm{M}$ unlabelled herring-sperm DNA. (c) To establish that the $\mathrm{His}_{6}$-tag did not contribute to altered migration of DNA targets, an enterokinase cleavage reaction was used to generate untagged Srv. The species of protein added to each lane is designated at the top (NP, no protein control). A representative EMSA with the probe spy0285 is shown.

for each probe and included 6000-fold molar excess unlabelled specific or non-specific competitor DNA. In all four cases rSrv demonstrated specificity for the PrfAbox targets as evidenced by the inability of non-specific DNA to completely abrogate binding. In contrast, addition of specific self DNA eliminated the Srv shift of each labelled probe (Fig. 3b).

To further establish that the $\mathrm{His}_{6}$ tag used to purify rSrv was not responsible for the altered migration of target DNA in EMSA (Bockmann et al., 1996; Dickneite et al., 1998), an enterokinase cleavage reaction was used to generate untagged Srv. The untagged Srv retarded the mobility of each of the four DNA targets tested (Fig. 3c and data not shown). The absence of the tag resulted in faster-migrating DNA-protein complexes due to the change in the molecular mass. However, higher-order multimers were still observed with the tagless product, although less abundant. Given the cross-linking, gel filtration and EMSA results coupled with mutational analysis (presented below), and the difficulty in obtaining adequate quantities of untagged Srv, the tagged rSrv was used except when indicated.

Although purified preparations of rSrv were used in EMSAs, control supershift experiments with S-tag-specific monoclonal antibody were carried out to verify that complexes observed in these assays were due solely to rSrv-DNA interaction. The S-tag monoclonal antibody (Novagen) specifically binds to the S-tag present on the recombinant Srv protein. Addition of $3 \mu$ undiluted antibody to binding reactions resulted in a supershift of protein-DNA complexes (data not shown).

\section{Analysis of relative affinity of Srv for GAS chromosomal targets}

The above results show that Srv binds DNA with a measurable degree of specificity. To examine possible 
differences in the relative binding affinity of $\mathrm{rSrv}$ we used EMSA and pixel densitometry. Percentage shift was calculated for binding reactions conducted over a range of rSrv concentrations $(0,0.09,0.42,3.82,7.64,15.3$ and $30.6 \mu \mathrm{M})$ and the relative affinity $\left(R_{\mathrm{A}}\right)$ was calculated as the amount of rSrv required to shift $50 \%$ of the free DNA (modified from Riggs et al., 1971). Density of unbound DNA was computed and used to determine percentage shift as described in Methods.

Analyses of $R_{\mathrm{A}}$ values derived from these calculations indicate that there is a measurable but not significant difference in the relative affinity $($ mean $=2.40 \pm 0.43 \mu \mathrm{M})$ of rSrv for these targets (Table 1).

\section{Site-directed mutation analysis suggests a role for the HTH motif in DNA binding}

While the EMSA studies indicated that Srv does bind DNA, they did not address the role of the HTH in this interaction. Previous work identified two mutations in PrfA that eliminated the ability of PrfA to bind DNA, and two others that enhanced the affinity of PrfA for the PrfA-Box (Eiting et al., 2005; Herler et al., 2001; Ripio et al., 1997; Sheehan et al., 1996; Shetron-Rama et al., 2003; Vega et al., 2004). These residues (G145, G155, S184 and R188) are conserved in Srv and correspond to residues G160, G174, S203 and R207. The glycine residues are located between the HTH and the $\beta$-roll structure, while S203 and R207 are located within the predicted HTH (Fig. 4a). Site-directed mutagenesis was used to determine if mutation of these residues would alter the ability of Srv to bind DNA. Where possible, residues were mutated to correspond to those residues which conferred an altered phenotype in PrfA (Eiting et al., 2005; Herler et al., 2001; Ripio et al., 1997; Sheehan et al., 1996; Shetron-Rama et al., 2003; Vega et al., 2004). As determined by EMSA, purified $\mathrm{rSrv}_{\mathrm{G} 174 \mathrm{~S}}$ and $\mathrm{rSrv}_{\mathrm{S} 203 \mathrm{~A}}$ resulted in reduced ability to bind DNA while the $\mathrm{rSrv}_{\mathrm{R} 207 \mathrm{G}}$ mutation completely abolished binding (Fig. 4b). $\mathrm{rSrv}_{\mathrm{G} 160 \mathrm{~S}}$ did not present a difference in DNA-binding phenotype (data not shown). Of note, we also generated $\mathrm{rSrv}_{\mathrm{G} 93 \mathrm{~A}}$ in order to produce a mutation within the $\beta$-roll structure. This mutant also did not present a difference in DNA-binding phenotype (data not shown). Site-directed mutants appeared to dimerize as wild-type Srv.

Table 1. Relative affinity $\left(R_{\mathrm{A}}\right)$ of Srv protein for PrfA-box-like DNA

\begin{tabular}{|lc|}
\hline Target DNA & $\boldsymbol{R}_{\mathbf{A}}^{*}(\boldsymbol{\mu M})$ \\
\hline spy0044 & $1.78 \pm 0.47$ \\
spy0285 & $2.72 \pm 0.72$ \\
spy0714 & $2.44 \pm 0.26$ \\
slr & $2.66 \pm 0.18$ \\
\hline
\end{tabular}

${ }^{*} R_{\mathrm{A}}$ was calculated as the amount of $\mathrm{rSrv}$ required to shift $50 \%$ of the free DNA.
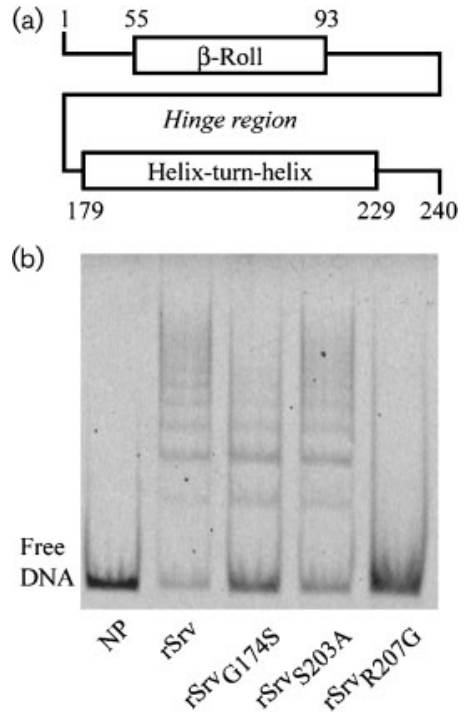

Fig. 4. Site-directed mutagenesis provides evidence that the HTH motif is involved in Srv-DNA interaction. (a) Diagram of Srv showing the amino acid locations of the $\beta$-roll structure, the hinge region and the HTH motif. Mutations were made to G174, S203 and R207 as detailed in the text. (b) $\mathrm{rSrv}(11 \mu \mathrm{M})$ was added to binding reactions with FAM-labelled target spy0285. As evidenced by changes in the concentration of free DNA between the no protein (NP) control and the experimental samples, each mutant bound less spy0285 than rSrv. $\mathrm{rSrv}_{\mathrm{R} 207 \mathrm{G}}$ was unable to retard the migration of spy0285. The EMSA image shown is representative of results obtained with targets spy0044, spy0714 and slr.

Loss of binding for $\mathrm{rSrv}_{\mathrm{R} 207 \mathrm{G}}$ and reduced binding of

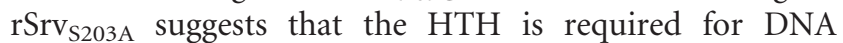
binding. Mutants $\mathrm{rSrv}_{\mathrm{G} 174 \mathrm{~S}}$ and $\mathrm{rSrv}_{\mathrm{S} 203 \mathrm{~A}}$ had $R_{\mathrm{A}}$ values that were 5.90 and 3.10 fold higher than that of rSrv bound to spy0285 (Fig. 5). Taken together, these data further support the hypothesis that the Srv HTH is a mediator of DNA interaction.

\section{Complementation with srv in trans restores wild- type SpeB activity while rSrv $_{\mathrm{R} 207 \mathrm{G}}$ does not}

Previously, we observed increased production and activity of SpeB in the $\Delta s r v$ strain (Reid et al., 2006). To test the hypothesis that this phenotype was due to the loss of Srv, we complemented srv in trans on the vector pIA $\beta 8$ and used a casein agar assay to test for SpeB proteolytic activity. The zone of proteolysis observed in the wild-type strain was restored in MGAS5005 $\Delta r r v$ (pIA $\beta 8$-srv) (Fig. 6). We believe this is an indirect effect of srv inactivation, and the intermediate(s) through which Srv is acting has/have not yet been identified (Reid et al., 2006). However, if the HTH of Srv is necessary for Srv function, then complementation of $\Delta s r v$ with the $s r v_{R 207 G}$ site-directed mutant should fail to restore that wild-type SpeB phenotype. Indeed, the strain 


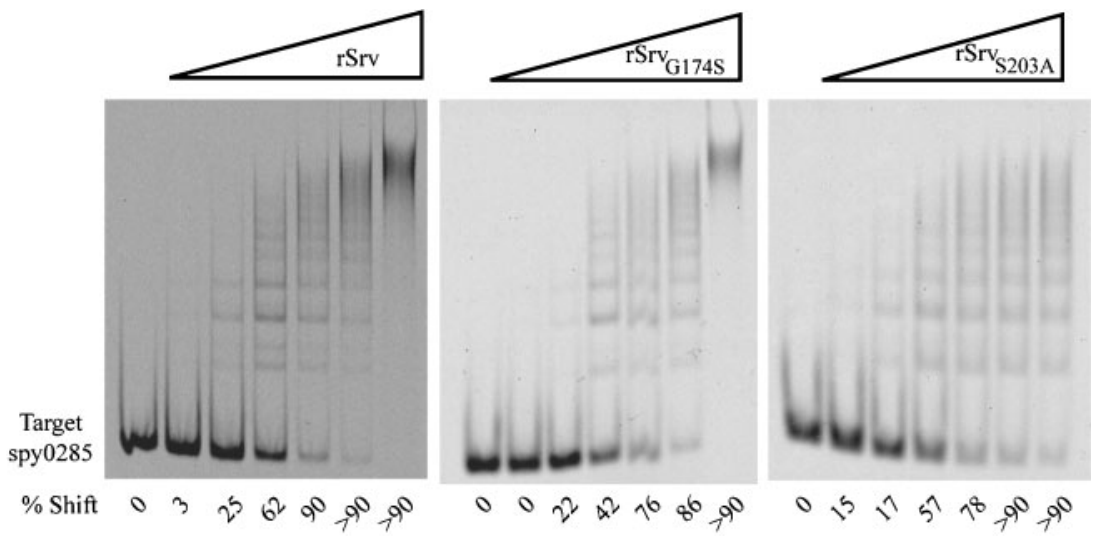

Fig. 5. EMSA titration of site-directed mutants. Purified $\mathrm{rSrv}_{\mathrm{G} 174 \mathrm{~S}}$ and $\mathrm{rSrv}_{\mathrm{S} 203 \mathrm{~A}}$ binding reactions were conducted with an increasing concentration of protein in order to determine the amount needed to retard the migration of $50 \%$ of the probe. Protein concentrations of $0,0.084,0.42,3.81,7.63$, 15.2 and $30.6 \mu \mathrm{M}$ were used. Percentage shift was calculated via pixel densitometry, rounded to the nearest whole per cent, and plotted to determine the relative affinity. $\mathrm{rSrv}_{\mathrm{R} 207 \mathrm{G}}$ did not bind DNA and was omitted from the analysis.

MGAS5005 $\Delta s r\left(\right.$ pIA $\left.\beta 8-s r v_{R 207 G}\right)$ produced the same enhanced zone of SpeB proteolysis as observed in MGAS5005 srv, suggesting that the HTH is not only required for DNA binding, but also contributes to Srv function.

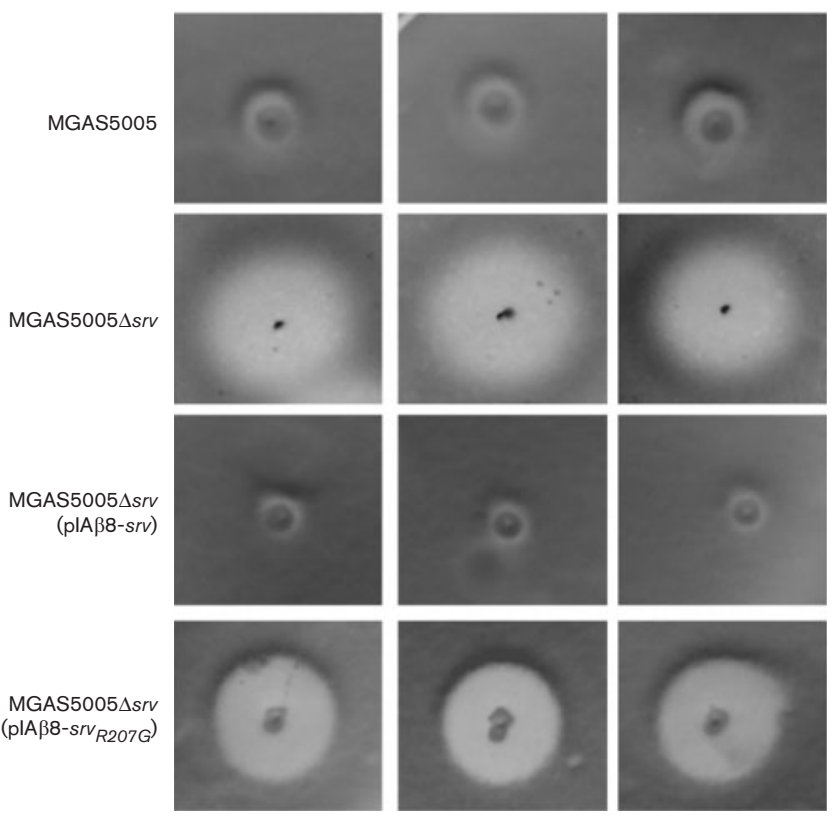

Fig. 6. Casein agar assay for SpeB proteolytic activity. MGAS5005, MGAS5005 $\Delta s r v$, MGAS5005 $\Delta s r v(p I A \beta 8-s r v)$ and MGAS5005 $\Delta s r\left(p I A \beta 8-s_{R 2} 207 G\right)$ were stab inoculated into agar plates containing skim milk and incubated for $18 \mathrm{~h}$ anaerobically. Proteinase activity is manifest as a zone of translucence surrounding the stab sites. There is a marked increase in the amount of SpeB proteolysis exhibited by MGAS5005 $\Delta s r v$ compared to MGAS5005. The wild-type phenotype is restored upon complementation of srv in trans [MGAS5005 $\Delta$ srv(pIA $\beta 8$ $s r v)]$. However, the $\Delta s r v$ phenotype is retained despite complementation with the $\operatorname{srv}_{R 207 G}$ allele [MGAS5005 $\Delta \operatorname{srv}(\mathrm{plA} \beta 8$ $\operatorname{srv}_{R 207 G)] \text {. }}$

\section{DISCUSSION}

$s r v$ encodes two putative structures characteristic of the CRP/FNR family: an N-terminal $\beta$-roll structure and a Cterminal HTH motif. In this regulatory family, the HTH motif has been shown to bind DNA-recognition sequences located in the proximity of genes under control of the regulator in question. However, the study of the CRP/FNR family has primarily taken place in Gram-negative organisms. In the case of PrfA, the best-studied CRP/FNR member of a Gram-positive pathogen, the regulator contributes to the virulence of an intracellular pathogen (Bubert et al., 1999; Mengaud et al., 1991). Given that GAS is an extracellular pathogen and the targets of Srv regulation are likely to be different from other species, we felt it necessary to test the hypothesis that the HTH of Srv binds DNA, and to study that mechanism.

As an initial test of this hypothesis, EMSAs were used to examine the ability of Srv to retard the migration of four selected DNA targets. EMSA experiments which contained $11.47 \mu \mathrm{M}$ rSrv bound and shifted $>90 \%$ of $6.7 \mathrm{nM}$ target DNA. However, the results showed a ladder-like banding pattern. Other DNA-binding regulators, such as SarA and SarZ of Staphylococcus aureus, have exhibited similar multibanding patterns (Kaito et al., 2006; Rechtin et al., 1999; Sterba et al., 2003). Morfeldt et al. (1996) presented evidence that suggested the banding pattern was due to SarA altering the superhelicity of the bound DNA. Under this assumption the ladder-like effect would be due to a single protein-DNA interaction yielding different conformations of varying electrophoretic mobility. Conversely, Kaito et al. (2006) observed a ladder-like pattern in SarZ binding, but speculated that the protein's ability to polymerize along the DNA was responsible for the multiple bands. In the same study, SarZ failed to demonstrate specific binding, as salmon-sperm competed equally with putative SarZ-regulated promoters of hla, agr and asp23 (Kaito et al., 2006). These data suggest that under the conditions tested the ladder-like banding pattern of SarZ was due to multiple non-specific binding events along the DNA (Kaito et al., 2006). To address this issue with Srv we conducted competition EMSAs using sonicated herring- 
sperm DNA as a non-specific competitor (Fig. 3). In these experiments, 6000-fold molar excess of herring-sperm was unable to compete away the Srv-DNA interaction while addition of equal amounts of unlabelled target DNA abolished retarded migration completely. These data suggest that Srv demonstrates a greater affinity for these targets than for non-specific DNA. Furthermore, they suggest that the multiple banding patterns we observed in competition experiments may be due to the specific interaction between Srv molecules rather than multiple Srv molecules binding non-specifically along the DNA.

CRP structure-function studies have revealed precise amino acid:DNA contact points present within the HTH (Ebright et al., 1984b; Parkinson et al., 1996; Zhang \& Ebright, 1990). These studies indicate that CRP residues R180, E181 and R185 form hydrogen bonds with DNA (Ebright et al., 1984a; Parkinson et al., 1996; Zhang \& Ebright, 1990). Similarly, mutation of PrfA residues S183, S184 and R188, which align with R180, E181 and R185 of CRP, disrupts the ability of PrfA to bind DNA (Herler et al., 2001; Sheehan et al., 1996). To test the hypothesis that Srv binds DNA via interaction with the HTH motif we selected Srv residues S203 and R207 (analogous to S184 and R188 of PrfA) for site-directed mutagenesis. In each case, residues were mutated to match the mutations that were observed to result in decreased binding of PrfA. While $\mathrm{rSrv}_{\mathrm{S} 203 \mathrm{~A}}$ did retain some ability to bind DNA, this ability

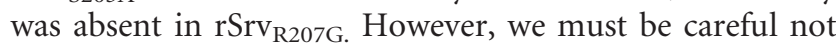
to assume that the HTH is necessary for Srv function. It may be that the regulatory effects of Srv are independent of DNA binding. To study this possibility, we complemented MGAS5005 $\Delta r v$ with $s r v_{R 207 G}$ in trans. We found that this construct was unable to restore the activity of the cysteine protease SpeB to wild-type levels. In contrast, complementation with srv returned SpeB production to expected levels. Thus, the HTH appears to be necessary for DNA binding and this likely contributes to Srv function. We speculate that R207 is a critical residue for Srv-DNA interaction and further hypothesize that it may be critical throughout Gram-positive members of the CRP/FNR family. Further support of this hypothesis is provided by an amino acid alignment of 20 putative Gram-positive CRP/FNR family members which indicated that R207 (CRP R185, PrfA R188) was $90 \%$ conserved (data not shown).

The focus of this work was to investigate the role of the Srv HTH in binding DNA. However, a brief discussion on the presence of the $\mathrm{N}$-terminal $\beta$-roll structure in Srv is warranted. Perhaps the best-studied example of this structure is in the activation of CRP by the effector molecule cAMP. Binding of cAMP to the $\beta$-roll structure results in an allosteric shift in the DNA-binding $\mathrm{HTH}$ motif which leads to an increase in DNA-binding affinity for specific CRP-dependent targets (Baichoo \& Heyduk, 1997; Garges \& Adhya, 1988; Harman, 2001; Heyduk \& Heyduk, 1994). While an analogous molecule has not been identified, and cAMP is not present at detectable levels in Gram-positive organisms, it is suspected that PrfA is activated by a signalling effector molecule (Hueck \& Hillen, 1995). Support for this hypothesis was demonstrated by a naturally occurring G145S mutation which results in activation of PrfA as measured by increased DNA-binding affinity and an enhanced ability to promote transcription (Ripio et al., 1997; Vega et al., 2004). In fact, $\operatorname{PrfA}_{\mathrm{G} 145 \mathrm{~S}}$ or $\operatorname{PrfA}^{\star}$ demonstrates an 18fold lower dissociation constant $(50 \mathrm{nM})$ than wild-type PrfA (Eiting et al., 2005). A number of mutations have now been identified that result in either $\mathrm{CRP}^{\star}$ or $\mathrm{PrfA}^{\star}$ phenotypes and most are located within the region between the $\beta$-roll structure and the HTH (Garges \& Adhya, 1985; Harman et al., 1986; Kolb et al., 1993; Vega et al., 1998, 2004). It is believed these mutations alter protein conformation, forcing the HTH into a position that is more conducive to DNA binding (Harman et al., 1986; Vega et al., 2004). We questioned whether a change within the same region in Srv would result in a Srv ${ }^{\star}$-like phenotype. Two amino acids were targeted for analysis: G174 and G160. These residues correspond to PrfA G155 and CRP G141, respectively. Mutation of these residues in PrfA to a serine resulted in *-like activity as measured by an increase in DNA-binding affinity in their respective backgrounds (Garges \& Adhya, 1988; Shetron-Rama et al., 2003). While G160S did not result in a change in rSrv DNA-binding affinity (data not shown), gel-shift experiments testing the ability of $\mathrm{rSrv}_{\mathrm{G} 174 \mathrm{~s}}$ to bind DNA showed that it actually bound with a lower affinity than $\mathrm{rSrv}_{\mathrm{WT}}$. Thus rather than generating an allosteric change resulting in a more favourable conformation for the $\mathrm{HTH}, \operatorname{Srv}_{\mathrm{G} 174 \mathrm{~S}}$ may have reduced access to the HTH. Another possibility is that while we have identified four targets which have allowed us to establish that Srv does interact with DNA through the $\mathrm{HTH}$, these may not be specific targets of Srv. Thus the mutations we have generated may result in a Srv${ }^{\star}$-like phenotype when tested against the proper target.

In conclusion, these data support our initial hypotheses that Srv binds DNA through a C-terminal HTH motif, and that this HTH contributes to Srv function. Questions still remain regarding the potential scope of the Srv regulon and the possible function of the $\mathrm{N}$-terminal $\beta$-roll structure. It is easy to hypothesize that $\mathrm{Srv}$ is a transcriptional regulator, given its homology to the CRP/ FNR family, its effect on virulence and its effect on gene transcription. However, it may function to facilitate the interaction of other regulators with their targets or aid in polymerase binding. PrfA has been shown to complex with RNA polymerase (Bockmann et al., 2000). With our new insight into the function of the $\mathrm{HTH}$, reporter systems can now be generated to directly test the ability of Srv to affect gene transcription. In addition, we are currently employing both a systematic evolution of ligands by exponential enrichment (SELEX) approach (Tuerk \& Gold, 1990) and in vivo chromatin immunoprecipitation to define potential Srv-dependent targets. 


\section{ACKNOWLEDGEMENTS}

This project was supported by Public Health Service Grant R01AI063453 from the National Institutes of Health to S.D. R. We thank D. J. Wozniak and W. E. Swords for critical reading of the manuscript as well as W. Debinski and J. Wykosky for assistance in gel filtration analysis and Kevin McIver for pIA $\beta 8$.

\section{REFERENCES}

Baichoo, N. \& Heyduk, T. (1997). Mapping conformational changes in a protein: application of a protein footprinting technique to cAMP-induced conformational changes in cAMP receptor protein. Biochemistry 36, 10830-10836.

Banks, D. J., Porcella, S. F., Barbian, K. D., Beres, S. B., Philips, L. E., Voyich, J. M., DeLeo, F. R., Martin, J. M., Somerville, G. A. \& Musser, J. M. (2004). Progress toward characterization of the group A Streptococcus metagenome: complete genome sequence of a macrolide-resistant serotype M6 strain. J Infect Dis 190, 727-738.

Beres, S. B., Sylva, G. L., Sturdevant, D. E., Granville, C. N., Liu, M., Ricklefs, S. M., Whitney, A. R., Parkins, L. D., Hoe, N. P. \& other authors (2004). Genome-wide molecular dissection of serotype M3 group A Streptococcus strains causing two epidemics of invasive infections. Proc Natl Acad Sci U S A 101, 11833-11838.

Blevins, J. S., Gillaspy, A. F., Rechtin, T. M., Hurlburt, B. K. \& Smeltzer, M. S. (1999). The staphylococcal accessory regulator (sar) represses transcription of the Staphylococcus aureus collagen adhesin gene (cna) in an agr-independent manner. Mol Microbiol 33, 317-326.

Bockmann, R., Dickneite, C., Middendorf, B., Goebel, W. \& Sokolovic, Z. (1996). Specific binding of the Listeria monocytogenes transcriptional regulator PrfA to target sequences requires additional factor(s) and is influenced by iron. Mol Microbiol 22, 643-653.

Bockmann, R., Dickneite, C., Goebel, W. \& Bohne, J. (2000). PrfA mediates specific binding of RNA polymerase of Listeria monocytogenes to PrfA-dependent virulence gene promoters resulting in a transcriptionally active complex. Mol Microbiol 36, 487-497.

Bohne, J., Kestler, H., Uebele, C., Sokolovic, Z. \& Goebel, W. (1996). Differential regulation of the virulence genes of Listeria monocytogenes by the transcriptional activator PrfA. Mol Microbiol 20, 1189-1198.

Bubert, A., Sokolovic, Z., Chun, S. K., Papatheodorou, L., Simm, A. \& Goebel, W. (1999). Differential expression of Listeria monocytogenes virulence genes in mammalian host cells. Mol Gen Genet 261, 323336.

Cunningham, M. W. (2000). Pathogenesis of group A streptococcal infections. Clin Microbiol Rev 13, 470-511.

Dickneite, C., Bockmann, R., Spory, A., Goebel, W. \& Sokolovic, Z. (1998). Differential interaction of the transcription factor PrfA and the PrfA-activating factor (Paf) of Listeria monocytogenes with target sequences. Mol Microbiol 27, 915-928.

Dramsi, S., Kocks, C., Forestier, C. \& Cossart, P. (1993). Internalinmediated invasion of epithelial cells by Listeria monocytogenes is regulated by the bacterial growth state, temperature and the pleiotropic activator prfA. Mol Microbiol 9, 931-941.

Ebright, R. H., Cossart, P., Gicquel-Sanzey, B. \& Beckwith, J. (1984a). Molecular basis of DNA sequence recognition by the catabolite gene activator protein: detailed inferences from three mutations that alter DNA sequence specificity. Proc Natl Acad Sci U S A 81, 7274-7278.

Ebright, R. H., Cossart, P., Gicquel-Sanzey, B. \& Beckwith, J. (1984b). Mutations that alter the DNA sequence specificity of the catabolite gene activator protein of E. coli. Nature 311, 232-235.
Eiting, M., Hageluken, G., Schubert, W. D. \& Heinz, D. W. (2005). The mutation G145S in PrfA, a key virulence regulator of Listeria monocytogenes, increases DNA-binding affinity by stabilizing the HTH motif. Mol Microbiol 56, 433-446.

Ferretti, J. J., McShan, W. M., Ajdic, D., Savic, D. J., Savic, G., Lyon, K., Primeaux, C., Sezate, S., Suvorov, A. N. \& other authors (2001). Complete genome sequence of an M1 strain of Streptococcus pyogenes. Proc Natl Acad Sci U S A 98, 4658-4663.

Freitag, N. E., Rong, L. \& Portnoy, D. A. (1993). Regulation of the prfA transcriptional activator of Listeria monocytogenes: multiple promoter elements contribute to intracellular growth and cell-to-cell spread. Infect Immun 61, 2537-2544.

Garges, S. \& Adhya, S. (1985). Sites of allosteric shift in the structure of the cyclic AMP receptor protein. Cell 41, 745-751.

Garges, S. \& Adhya, S. (1988). Cyclic AMP-induced conformational change of cyclic AMP receptor protein (CRP): intragenic suppressors of cyclic AMP-independent CRP mutations. J Bacteriol 170, 14171422 .

Graham, M. R., Smoot, L. M., Migliaccio, C. A., Virtaneva, K., Sturdevant, D. E., Porcella, S. F., Federle, M. J., Adams, G. J., Scott, J. R. \& Musser, J. M. (2002). Virulence control in group A Streptococcus by a two-component gene regulatory system: global expression profiling and in vivo infection modeling. Proc Natl Acad Sci U S A 99, 13855-13860.

Harman, J. G. (2001). Allosteric regulation of the cAMP receptor protein. Biochim Biophys Acta 1547, 1-17.

Harman, J. G., McKenney, K. \& Peterkofsky, A. (1986). Structurefunction analysis of three cAMP-independent forms of the cAMP receptor protein. J Biol Chem 261, 16332-16339.

Herler, M., Bubert, A., Goetz, M., Vega, Y., Vazquez-Boland, J. A. \& Goebel, W. (2001). Positive selection of mutations leading to loss or reduction of transcriptional activity of PrfA, the central regulator of Listeria monocytogenes virulence. J Bacteriol 183, 5562-5570.

Heyduk, E. \& Heyduk, T. (1994). Mapping protein domains involved in macromolecular interactions: a novel protein footprinting approach. Biochemistry 33, 9643-9650.

Hueck, C. J. \& Hillen, W. (1995). Catabolite repression in Bacillus subtilis: a global regulatory mechanism for the Gram-positive bacteria? Mol Microbiol 15, 395-401.

Hynes, W. L. \& Tagg, J. R. (1985). A simple plate assay for detection of group A streptococcus proteinase. J Microbiol Methods 4, 25-31.

Kaito, C., Morishita, D., Matsumoto, Y., Kurokawa, K. \& Sekimizu, K. (2006). Novel DNA binding protein SarZ contributes to virulence in Staphylococcus aureus. Mol Microbiol 62, 1601-1617.

Kolb, A., Busby, S., Buc, H., Garges, S. \& Adhya, S. (1993). Transcriptional regulation by cAMP and its receptor protein. Annu Rev Biochem 62, 749-795.

Kreikemeyer, B., Mclver, K. S. \& Podbielski, A. (2003). Virulence factor regulation and regulatory networks in Streptococcus pyogenes and their impact on pathogen-host interactions. Trends Microbiol 11, 224-232.

Kuusinen, A., Abele, R., Madden, D. R. \& Keinanen, K. (1999) Oligomerization and ligand-binding properties of the ectodomain of the alpha-amino-3-hydroxy-5-methyl-4-isoxazole propionic acid receptor subunit GluRD. J Biol Chem 274, 28937-28943.

Mengaud, J., Vicente, M. F. \& Cossart, P. (1989). Transcriptional mapping and nucleotide sequence of the Listeria monocytogenes hlyA region reveal structural features that may be involved in regulation. Infect Immun 57, 3695-3701.

Mengaud, J., Dramsi, S., Gouin, E., Vazquez-Boland, J. A., Milon, G. \& Cossart, P. (1991). Pleiotropic control of Listeria monocytogenes 
virulence factors by a gene that is autoregulated. Mol Microbiol 5, 2273-2283.

Morfeldt, E., Tegmark, K. \& Arvidson, S. (1996). Transcriptional control of the agr-dependent virulence gene regulator, RNAIII, in Staphylococcus aureus. Mol Microbiol 21, 1227-1237.

Musser, J. M. \& DeLeo, F. R. (2005). Toward a genome-wide systems biology analysis of host-pathogen interactions in group A Streptococcus. Am J Pathol 167, 1461-1472.

Parkinson, G., Wilson, C., Gunasekera, A., Ebright, Y. W., Ebright, R. E. \& Berman, H. M. (1996). Structure of the CAP-DNA complex at 2.5 angstroms resolution: a complete picture of the protein-DNA interface. J Mol Biol 260, 395-408.

Passner, J. M., Schultz, S. C. \& Steitz, T. A. (2000). Modeling the cAMP-induced allosteric transition using the crystal structure of CAP-cAMP at $2.1 \AA$ resolution. J Mol Biol 304, 847-859.

Pérez-Arellano, I., Zúñiga, M. \& Pérez-Martínez, G. (2001). Construction of compatible wide-host-range shuttle vectors for lactic acid bacteria and Escherichia coli. Plasmid 46, 106-116.

Ramsey, D. M., Baynham, P. J. \& Wozniak, D. J. (2005). Binding of Pseudomonas aeruginosa AlgZ to sites upstream of the algZ promoter leads to repression of transcription. J Bacteriol 187, 4430-4443.

Rechtin, T. M., Gillaspy, A. F., Schumacher, M. A., Brennan, R. G., Smeltzer, M. S. \& Hurlburt, B. K. (1999). Characterization of the SarA virulence gene regulator of Staphylococcus aureus. Mol Microbiol 33, 307-316.

Reid, S. D., Hoe, N. P., Smoot, L. M. \& Musser, J. M. (2001). Group A Streptococcus: allelic variation, population genetics, and hostpathogen interactions. J Clin Invest 107, 393-399.

Reid, S. D., Montgomery, A. G. \& Musser, J. M. (2004). Identification of $s r v$, a PrfA-like regulator of group A streptococcus that influences virulence. Infect Immun 72, 1799-1803.

Reid, S. D., Chaussee, M. S., Doern, C. D., Chaussee, M. A., Montgomery, A. G., Sturdevant, D. E. \& Musser, J. M. (2006). Inactivation of the group A Streptococcus regulator srv results in chromosome wide reduction of transcript levels, and changes in extracellular levels of Sic and SpeB. FEMS Immunol Med Microbiol 48, 283-292.

Riggs, A. D., Reiness, G. \& Zubay, G. (1971). Purification and DNAbinding properties of the catabolite gene activator protein. Proc Natl Acad Sci U S A 68, 1222-1225.

Ripio, M. T., Dominguez-Bernal, G., Lara, M., Suarez, M. \& VazquezBoland, J. A. (1997). A Gly145Ser substitution in the transcriptional activator PrfA causes constitutive overexpression of virulence factors in Listeria monocytogenes. J Bacteriol 179, 1533-1540.

Schultz, S. C., Shields, G. C. \& Steitz, T. A. (1990). Crystallization of Escherichia coli catabolite gene activator protein with its DNA binding site. The use of modular DNA. J Mol Biol 213, 159-166.

Schultz, S. C., Shields, G. C. \& Steitz, T. A. (1991). Crystal structure of a CAP-DNA complex: the DNA is bent by 90 degrees. Science 253, 1001-1007.
Sheehan, B., Klarsfeld, A., Msadek, T. \& Cossart, P. (1995). Differential activation of virulence gene expression by PrfA, the Listeria monocytogenes virulence regulator. J Bacteriol 177, 6469-6476.

Sheehan, B., Klarsfeld, A., Ebright, R. \& Cossart, P. (1996). A single substitution in the putative helix-turn-helix motif of the pleiotropic activator PrfA attenuates Listeria monocytogenes virulence. Mol Microbiol 20, 785-797.

Shetron-Rama, L. M., Mueller, K., Bravo, J. M., Bouwer, H. G., Way, S. S. \& Freitag, N. E. (2003). Isolation of Listeria monocytogenes mutants with high-level in vitro expression of host cytosol-induced gene products. Mol Microbiol 48, 1537-1551.

Smoot, J. C., Barbian, K. D., Van Gompel, J. J., Smoot, L. M., Chaussee, M. S., Sylva, G. L., Sturdevant, D. E., Ricklefs, S. M., Porcella, S. F. \& other authors (2002). Genome sequence and comparative microarray analysis of serotype M18 group A Streptococcus strains associated with acute rheumatic fever outbreaks. Proc Natl Acad Sci U S A 99, 4668-4673.

Sterba, K. M., Mackintosh, S. G., Blevins, J. S., Hurlburt, B. K. \& Smeltzer, M. S. (2003). Characterization of Staphylococcus aureus SarA binding sites. J Bacteriol 185, 4410-4417.

Tuerk, C. \& Gold, L. (1990). Systematic evolution of ligands by exponential enrichment: RNA ligands to bacteriophage T4 DNA polymerase. Science 249, 505-510.

Vega, Y., Dickneite, C., Ripio, M. T., Bockmann, R., Gonzalez-Zorn, B., Novella, S., Dominguez-Bernal, G., Goebel, W. \& Vazquez-Boland, J. A. (1998). Functional similarities between the Listeria monocytogenes virulence regulator PrfA and cyclic AMP receptor protein: the PrfA* (Gly145Ser) mutation increases binding affinity for target DNA. J Bacteriol 180, 6655-6660.

Vega, Y., Rauch, M., Banfield, M. J., Ermolaeva, S., Scortti, M., Goebel, W. \& Vazquez-Boland, J. A. (2004). New Listeria monocytogenes prfA $A^{\star}$ mutants, transcriptional properties of $\operatorname{PrfA}^{\star}$ proteins and structure-function of the virulence regulator PrfA. Mol Microbiol 52, 1553-1565.

Velge, P., Herler, M., Johansson, J., Roche, S. M., Temoin, S., Fedorov, A. A., Gracieux, P., Almo, S. C., Goebel, W. \& Cossart, P. (2007). A naturally occurring mutation K220T in the pleiotropic activator PrfA of Listeria monocytogenes results in a loss of virulence due to decreasing DNA-binding affinity. Microbiology 153, 995-1005.

Weber, I. T. \& Steitz, T. A. (1987). Structure of a complex of catabolite gene activator protein and cyclic AMP refined at $2.5 \AA$ resolution. J Mol Biol 198, 311-326.

Zhang, X. P. \& Ebright, R. H. (1990). Identification of a contact between arginine-180 of the catabolite gene activator protein (CAP) and base pair 5 of the DNA site in the CAP-DNA complex. Proc Natl Acad Sci U S A 87, 4717-4721.

Edited by: T. J. Mitchell 